\title{
ON A SEMILINEAR VARIATIONAL PROBLEM
}

\author{
BERND SCHMIDT ${ }^{1}$
}

\begin{abstract}
We provide a detailed analysis of the minimizers of the functional $u \mapsto \int_{\mathbb{R} n}|\nabla u|^{2}+$ $D \int_{\mathbb{R}^{n}}|u|^{\gamma}, \gamma \in(0,2)$, subject to the constraint $\|u\|_{L^{2}}=1$. This problem, e.g., describes the longtime behavior of the parabolic Anderson in probability theory or ground state solutions of a nonlinear Schrödinger equation. While existence can be proved with standard methods, we show that the usual uniqueness results obtained with PDE-methods can be considerably simplified by additional variational arguments. In addition, we investigate qualitative properties of the minimizers and also study their behavior near the critical exponent 2 .
\end{abstract}

Mathematics Subject Classification. 35J20, 49J45, 35Q55.

Received February 22, 2009. Revised July 27, 2009.

Published online October 9, 2009.

\section{IntroduCtion AND STATEMENT OF THE MAIN RESUltS}

Consider the variational problems

$$
I(u)=I_{\gamma, D}(u):=\int_{\mathbb{R}^{n}}|\nabla u|^{2}+D \int_{\mathbb{R}^{n}}|u|^{\gamma} \rightarrow \min
$$

for $0<\gamma<2, D>0$ and $u$ in the class of admissible functions

$$
\mathcal{A}:=\left\{u \in H^{1}\left(\mathbb{R}^{n}\right):\|u\|_{L^{2}}=1\right\} .
$$

Due to the constraint $\|u\|_{L^{2}}=1$, even for $\gamma \geq 1$ this is a non-convex minimization problem. We will study the existence, uniqueness and qualitative aspects of minimizers of (1.1).

Our main motivation for this investigation comes from the long-time behavior of the parabolic Anderson model

$$
\left\{\begin{aligned}
\frac{\partial}{\partial t} v(z, t) & =\Delta^{n} v(z, t)+\xi(z) v(z, t), & & t \in(0, \infty), z \in \mathbb{Z}^{n}, \\
v(z, 0) & =\delta_{0}(z), & & z \in \mathbb{Z}^{n} .
\end{aligned}\right.
$$

Here $(\xi(z))_{z \in \mathbb{Z}^{n}}$ is a family of i.i.d. random variables bounded from above and $\Delta^{n}$ denotes the discrete Laplacian $\Delta^{n} v(z)=\sum_{x \in \mathbb{Z}^{n}:|x-z|=1}(f(x)-f(z))$. We refer to [8] for more background information on the parabolic Anderson model. In [2] a variational formula is derived for the long-time asymptotics of the total mass in this system in terms of the minimal value of $I$. Here the constants $\gamma$ and $D$ describe the limiting behavior

Keywords and phrases. Nonlinear minimum problem, parabolic Anderson model, variational methods, Gamma-convergence, ground state solutions.

1 Zentrum Mathematik, Technische Universität München, Boltzmannstr. 3, 85747 Garching, Germany. schmidt@ma.tum.de 
of the density of the distribution of $\xi(z)$ near its essential supremum. The minimizers - if they exist - can be interpreted as the shape of suitably rescaled solutions on certain regions which contribute in an optimal way to the total mass within the system (see [2,9] for more details). It is therefore interesting to also investigate the minimizers rather than only the minimal values of this functional ${ }^{2}$.

As a perhaps more prominent example where problems of this type occur we also mention the theory of nonlinear Schrödinger equations (cf. e.g. [1]). If $I$ as in (1.1) describes a nonlinear quantum Hamiltonian, one seeks for ground states of the system, that is for minimizers of $I$.

A lot of information can be obtained by studying the associated Euler-Lagrange equation of (1.1). In fact, there exists an abundant literature on semilinear Dirichlet problems of the form

$$
\Delta u+f(u)=0
$$

and there are existence, uniqueness and symmetry results under various assumptions on the nonlinearity $f$ for positive solutions of (1.2) decaying to 0 at infinity. We do not give a complete account on the literature here but just refer to $[6,7,12]$ and the references therein, which will be of particular interest to our situation.

Note, however, that in both examples discussed above, $u$ in addition is a minimizer of $I$. One of our main goals is to show that this additional feature greatly facilitates the arguments needed to analyze solutions of (1.2), even if one restricts to considering only radially symmetric and non-negative functions $u$. Also note that only for the variational problem solutions will in fact be radially symmetric (up to translations).

An outline of the paper is as follows. In Section 2 we will show that minimizers of $I$ exist and $I$ attains its minimal value at a radially symmetric and radially decreasing function. We mention that variational techniques of the type we will encounter here have been used before to establish existence of solutions of (1.2). On bounded domains, i.e. for the functionals $I_{\rho}$ restricted to functions supported on a ball of radius $\rho$, existence readily follows by standard methods. To obtain existence on $\mathbb{R}^{n}$ we will give a proof here that very naturally links the functionals $I_{\rho}$ to the limiting functional $I$ by $\Gamma$-convergence. A mild equicoercivity estimate then yields the desired result. (Note that this provides, e.g., an alternative proof for the existence statement in [6], which only uses elementary convexity arguments.)

In the following Section 3 we examine the Euler-Lagrange equation associated with $I$. A standard argument will imply that minimizers of $I$ are compactly supported and (possibly after switching sign and a translation) are radially symmetric and radially decreasing. (This shows that in fact minimizers of $I$ are also minimizers of $I_{\rho}$ for $\rho$ sufficiently large.) Also note that the symmetry result is a variational effect that cannot follow from the PDE alone, for if $u$ is any minimizer and $R$ sufficiently large, also $u+u(\cdot+R)$ is a solution of the Euler-Lagrange equation. We finally investigate the regularity properties of minimizers $u$. It easily follows that $u$ is $C^{\infty}$-smooth away from the sphere $\partial \operatorname{supp} u$, where $u$ becomes 0 . We also study the behavior of $u$ in the vicinity of this singular set in detail.

The greatest advantage of keeping a variational point of view is in the proof of uniqueness (up to translation and sign-changes) of minimizers, which will be given in Section 4. Having noted that all minimizers satisfy the same Euler-Lagrange equation, one could refer to uniqueness results as proved in [6,7,12]. However, an additional variational convexity argument will greatly simplify these approaches. As it turns out, the - due to the singularity - seemingly harder case $\gamma \leq 1$ becomes in fact much easier. The case $\gamma>1$, on the contrary, yields a convex non-linearity and is more difficult.

We summarize the main results of these sections in the following Theorem, whose proof directly follows from Corollary 2.6, Proposition 3.3, Lemma 3.2, Lemmas 4.1, 3.1 and Proposition 4.2.

Theorem 1.1. Suppose $I$ is given as in (1.1) with $D>0,0<\gamma<2$.

(i) There exist minimizers of $I$.

(ii) After a suitable translation and, possibly, a change of sign, every minimizer is non-negative, rotationally symmetric about the origin, decreasing in the radial variable and compactly supported.

(iii) The non-negative radially symmetric minimizer is unique.

\footnotetext{
${ }^{2}$ More precisely, the functional considered in [2] is $\inf _{\rho>0} I_{\rho}$, where $I_{\rho}=I_{\rho, \gamma, D}$ are the restrictions to $\mathcal{A}_{\rho}:=\{u \in \mathcal{A}: \operatorname{supp}(u) \in$ $\left.Q_{\rho}\right\}$ and the $Q_{\rho}$ can be taken as balls $\bar{B}_{\rho}$ of radius $\rho$. This is in fact equivalent to our original problem.
} 
The precise statement about the regularity of minimizers is given in Proposition 3.5.

Finally, in Section 5 we first discuss two cases where explicit formulae for the minimizers can be given. This is possible in one dimension for every $\gamma$ (as has been noted already by Biskup and König, unpublished notes) and in any dimension if the Euler-Lagrange equation becomes linear. Interestingly, there is also an explicit asymptotic formula for the minimizers when $\gamma$ approaches the critical exponent 2: if $\gamma \rightarrow 2$, then the corresponding minimizers $u_{\gamma}$ - suitably rescaled - converge to a Gaussian function with covariance matrix $\frac{1}{D} \mathrm{Id}$ :

$$
(2-\gamma)^{\frac{n}{4}} u_{\gamma}\left(\frac{x}{\sqrt{2-\gamma}}\right) \rightarrow\left(\frac{D}{2 \pi}\right)^{\frac{n}{2}} \mathrm{e}^{-\frac{D|x|^{2}}{2}} .
$$

See Propositions 5.1, 5.2 and Theorem 5.3 for the precise statement of these results.

For the sake of clarity we confine our analysis to the functional introduced in (1.1), but we believe that the variational point of view advocated here might be useful in more general situations. In fact, some of the arguments developed here are easily seen to apply in more general situations. (E.g., a straightforward adaption of the method showing existence gives an alternative proof of the existence theorem in [6]. Also, the particular form of the nonlinearity in the uniqueness proof is not relevant to our analysis.)

\section{Existence AND $\Gamma$-CONVERGENCE}

Our first aim is to obtain the existence of minimizers of $I$. We will do this by reducing to the functionals $I_{\rho}=I_{\rho, \gamma, D}$, the restriction of $I$ to $\mathcal{A}_{\rho}=\left\{u \in \mathcal{A}: \operatorname{supp} u \subset \bar{B}_{\rho}\right\}, B_{\rho}$ the ball of radius $\rho$ about the origin.

A standard application of the direct method gives the following

Lemma 2.1. Minimizers of $I_{\rho}, 0<\rho<\infty$, exist.

Proof. Choose a minimizing sequence $\left(u_{k}\right)$ such that $u_{k} \rightarrow u$ in $H^{1}\left(B_{\rho}\right)$. Since $u_{k} \rightarrow u \in \mathcal{A}$ in $L^{2}$ and $|u|^{\gamma} \rightarrow|u|^{\gamma}$ in $L^{\frac{2}{\gamma}}$, by lower semicontinuity of the gradient term we find $I_{\rho}(u) \leq \liminf I_{\rho}\left(u_{k}\right)=\inf I_{\rho}$.

The next task is to show that minimizers can be chosen to be radially decreasing, i.e, to only depend on the radial variable $r=|x| \in[0, \infty)$ and to be decreasing in $r$. If a function $f: \mathbb{R}^{n} \rightarrow \mathbb{R}$ decays to 0 at infinity in the sense that $|\{x:|f(x)|>t\}|<\infty$ for all $t>0$, its symmetrically decreasing rearrangement (or Schwarz symmetrization) is defined by

$$
f^{*}(x):=\sup \left\{t:|\{|f|>t\}|>\omega_{n}|x|^{n}\right\},
$$

where $\omega_{n}$ denotes the volume of the $n$-dimensional unit ball. (See, e.g., [11] for basic properties of symmetric rearrangements.) For easy reference we give the following lemma from [4]:

Lemma 2.2. Suppose $f: \mathbb{R}^{n} \rightarrow \mathbb{R}$ decays to 0 at infinity. Then, for any $\alpha>0$,

$$
\int|f|^{\alpha}(x) \mathrm{d} x=\int\left(f^{*}\right)^{\alpha}(x) \mathrm{d} x
$$

whenever one of these terms is finite. If $f \in H^{1}\left(\mathbb{R}^{n}\right)$, then also $f^{*} \in H^{1}\left(\mathbb{R}^{n}\right)$ and

$$
\int\left|\nabla f^{*}(x)\right|^{2} \mathrm{~d} x \leq \int|\nabla f(x)|^{2} \mathrm{~d} x
$$

Equality can occur in (2.1) only if $|f|=f^{*}(\cdot-a)$ for a suitable translation vector a under the assumption that $\mid\left\{\nabla f^{*}=0\right\} \cap\left(f^{*}\right)^{-1}\left(0\right.$, ess $\left.\sup f^{*}\right) \mid=0$, or, equivalently, $\frac{\mathrm{d}}{\mathrm{d} r} \tilde{f}^{*}(r) \neq 0$ for almost all $r$ such that $\tilde{f}^{*}(r) \in$ $\left(0, \operatorname{ess} \sup f^{*}\right)$, where $\tilde{f}^{*}$ is defined by $f^{*}(x)=\tilde{f} *(|x|)$.

Note that in the sequel we will not distinguish in our notation between $f$ and $\tilde{f}$ with $\tilde{f}(|x|)=f(x)$ for a radially symmetric function $f$. 
Lemma 2.3. There are radially decreasing minimizers of $I_{\rho}$.

Proof. Clear by Lemmas 2.2 and 2.1 .

We now turn to the problem of finding minimizers of $I$. More generally, we establish the following connection between $I_{\rho}$ and $I$. (See, e.g., [3] for a general introduction to the theory of $\Gamma$-convergence.)

Proposition 2.4. The functionals $I_{\rho}$ (extended by $+\infty$ outside $\mathcal{A}_{\rho}$ to all of $\mathcal{A}$ ) $\Gamma$-converge to $I$ with respect to the strong $L^{2}$-topology on $\mathcal{A}$.

Proof. Let $u \in \mathcal{A}$. By multiplication with suitable cut-off functions $\theta_{\rho}: \mathbb{R}^{n} \rightarrow[0,1]$ such that $\theta_{\rho} \equiv 1$ on $B_{\rho-1}$, $\theta_{\rho} \equiv 0$ on $\mathbb{R}^{n} \backslash B_{\rho}$ and $\left|\nabla \theta_{\rho}\right| \leq C$, we find $v_{\rho}=\theta_{\rho} u$, supported on $B_{\rho}$, such that $\left|v_{\rho}\right| \leq|u|$ and $v_{\rho} \rightarrow u$ in $H^{1}$. Then $u_{\rho}:=\frac{v_{\rho}}{\left\|v_{\rho}\right\|_{L^{2}}} \in \mathcal{A}$ is a recovery sequence for $u$ :

$$
\limsup _{\rho \rightarrow \infty} I_{\rho}\left(u_{\rho}\right)=\lim _{\rho \rightarrow \infty} \frac{1}{2}\left\|v_{\rho}\right\|_{L^{2}}^{-2} \int_{\mathbb{R}^{n}}\left|\nabla v_{\rho}\right|^{2}+\limsup _{\rho \rightarrow \infty} D\left\|v_{\rho}\right\|_{L^{2}}^{-\gamma} \int_{\mathbb{R}^{n}}\left|v_{\rho}\right|^{\gamma} \leq I(u) .
$$

To prove the $\Gamma$-liminf inequality, let $u_{\rho} \rightarrow u$ and without loss of generality assume that the sequence $\left(I_{\rho}\left(u_{\rho}\right)\right)$ is bounded, whence $u_{\rho} \rightarrow u$ in $H^{1}$. On every ball $B_{R}$ of radius $R>0$, as in the proof of Lemma 2.1, we see that

$$
\liminf _{\rho \rightarrow \infty} I_{\rho}\left(u_{\rho}\right) \geq \liminf _{\rho \rightarrow \infty}\left(\frac{1}{2} \int_{B_{R}}\left|\nabla u_{\rho}\right|^{2}+D \int_{B_{R}}\left|u_{\rho}\right|^{\gamma}\right) \geq \frac{1}{2} \int_{B_{R}}|\nabla u|^{2}+D \int_{B_{R}}|u|^{\gamma}
$$

Now sending $R \rightarrow \infty$ we obtain that indeed $\liminf _{\rho \rightarrow \infty} I_{\rho}\left(u_{\rho}\right) \geq I(u)$ by monotone convergence.

We now prove equi-mild coercivity for the functionals $I_{\rho}$ as $\rho \rightarrow \infty$.

Lemma 2.5. Let $\rho_{k} \rightarrow \infty$ and $\left(u_{k}\right)$ be a sequence of radially decreasing minimizers of $I_{\rho_{k}}$. Then for a subsequence (not relabeled) $u_{k} \rightarrow u$ in $L^{2}$ for some $u \in \mathcal{A}$.

Proof. Since the sequence $\left(I_{\rho_{k}}\left(u_{k}\right)\right)$ is bounded, by passing if necessary to a suitable subsequence we may assume that $u_{k} \rightarrow u$ in $H^{1}\left(\mathbb{R}^{n}\right)$ for some $u \in H^{1}\left(\mathbb{R}^{n}\right)$. It only remains to prove that $\|u\|_{L^{2}} \geq 1$.

Assume that $\|u\|_{L^{2}}<1-\varepsilon$ for some $\varepsilon>0$. Then for each ball $B_{R}$ there is $k_{0}(R)$ such that

$$
\left\|u_{k} \chi_{\mathbb{R}^{n} \backslash B_{R}}\right\|_{L^{2}} \geq \varepsilon \text { for } k \geq k_{0}
$$

because otherwise $1-\varepsilon \leq \lim \sup _{k \rightarrow \infty}\left\|u_{k} \chi_{B_{R}}\right\|_{L^{2}}=\left\|u \chi_{B_{R}}\right\|_{L^{2}} \leq\|u\|_{L^{2}}$ since $u_{k} \rightarrow u$ in $L^{2}$ on the bounded set $B_{R}$. (Here $\chi_{M}$ denotes the characteristic function of the set $M \subset \mathbb{R}^{n}$.) Now let $\delta>0$ and set $v_{k}:=u_{k}^{2} \chi_{\mathbb{R}^{n} \backslash B_{R}}$ for $R=\left(\omega_{n} \delta^{2}\right)^{-\frac{1}{n}}$. Since the $u_{k}$ are radially decreasing, we find that $0 \leq v_{k} \leq \delta^{2}$ for all $k \in \mathbb{N}$ and $\left\|v_{k}\right\|_{L^{1}} \geq \varepsilon^{2}$ for $k \geq k_{0}(R)$. Clearly, $I_{\rho_{k}}\left(u_{k}\right) \geq D \int_{\mathbb{R}^{n}} v_{k}^{\gamma / 2}$.

Now note that the variational problem

$$
\int_{\mathbb{R}^{n}} v^{\frac{\gamma}{2}} \rightarrow \min , \quad \text { subject to } 0 \leq v \leq \delta^{2} \text { and }\|v\|_{L^{1}}=c(\text { fixed })
$$

can be solved noting that $x \mapsto x^{\gamma / 2}$ is concave: An optimal choice is $v=\delta^{2} \chi_{M}$ for a set $M$ of measure $c \delta^{-2}$ with value $\int_{\mathbb{R}^{n}} v^{\frac{\gamma}{2}}=c \delta^{\gamma-2}$. It follows that $\liminf _{k \rightarrow \infty} I_{\rho_{k}}\left(u_{k}\right) \geq \varepsilon^{2} \delta^{\gamma-2}$ for all $\delta>0$ contradicting the boundedness of $\left(I_{\rho_{k}}\left(u_{k}\right)\right)$.

A standard argument in the theory of $\Gamma$-convergence now implies the existence of minimizers of $I$ :

Corollary 2.6. There exist radially decreasing minimizers of $I$. 
Proof. By Lemma 2.5 there exists a sequence $\left(u_{k}\right)$ of radially decreasing minimizers of $I_{\rho_{k}}$ such that $u_{k} \rightarrow u$ in $L^{2}$, whence also $u$ is radially decreasing. Then Proposition 2.4 shows that $I(u)=\inf I$ : If $v \in \mathcal{A}$, choosing a recovery sequence for $v$ we see that $I(v)=\lim _{k} I_{\rho_{k}}\left(v_{k}\right) \geq \liminf _{k \rightarrow \infty} I_{\rho_{k}}\left(u_{k}\right) \geq I(u)$.

This, in particular, proves Theorem 1.1(i).

\section{Qualitative PRoperties of Minimizers}

In this section we study the qualitative aspects of minimizers of $I$. We first derive the Euler-Lagrange equation and show that minimizers have compact support. Next we investigate the regularity and symmetry properties of minimizers.

Suppose $u$ is a radially decreasing minimizer of $I$ (or $I_{\rho}$ for $\left.\rho<\infty\right)$. By $B_{R}=B_{R(u)}$ we denote the open ball $B_{R}=\{u>0\}$ of radius $R \in(0, \infty]$, so that $u \in H_{0}^{1}\left(B_{R}\right) \cap C\left(\overline{B_{R}}\right)$.

Note that if $\gamma \leq 1$, then $I$ is not differentiable at $u$ in directions that do not vanish on $\{u=0\}$, so we first consider variations concentrated on $\{u>0\}$. Since inf $u>0$ on every compact subset of $B_{R}$, by standard methods we find that

$$
\int_{B_{R}} \nabla u \cdot \nabla v+D \gamma \int_{B_{R}}|u|^{\gamma-1} v-\lambda \int_{B_{R}} u v=0
$$

for all $v \in C_{c}^{\infty}\left(B_{R}\right)$ and some Lagrange-multiplier $\lambda=\lambda(u) \in \mathbb{R}$ (due to the side-condition $\int u^{2}=1$ ), so $u \in H_{0}^{1}\left(B_{R}\right)$ is a distributional solution of

$$
\Delta u+\lambda u-D \gamma|u|^{\gamma-1}=0 \quad \text { in } B_{R}
$$

Note that in fact (3.1) holds for all $v \in H_{0}^{1}\left(B_{R}\right)$. If $v$ has compact support in $B_{R}$ this follows from a standard approximation argument. If $v \geq 0$, it can be seen by using approximations $\theta_{k} v \rightarrow v$ in $H_{0}^{1}\left(B_{R}\right)$ with smooth cut-off function $\theta_{k}, k \in \mathbb{N}$, where $\theta_{k} \nearrow 1$ on $B_{R}$, and monotone convergence. For general $v$ one then splits $v$ into $v=v^{+}-v^{-}$.

By elliptic regularity, we have in fact $u \in C^{\infty}\left(B_{R}\right)$. Notice also that, as $u$ is radially symmetric, $|x|=: r \mapsto$ $u(r)$ solves

$$
\left\{\begin{aligned}
u^{\prime \prime}+\frac{n-1}{r} u^{\prime}+\lambda u-D \gamma|u|^{\gamma-1} & =0 & & \text { for } 0<r<R, \\
u(r) & =0 & & \text { for } r=R, \\
u^{\prime}(r) & =0 & & \text { for } r=0 .
\end{aligned}\right.
$$

Finally observe that $u^{\prime}<0$ on $(0, R)$, for if there were some $r_{1} \in(0, R)$ with $u^{\prime}\left(r_{1}\right)=0$, then also $u^{\prime \prime}\left(r_{1}\right)=0$ since $u$ decreases and so $\lambda u\left(r_{1}\right)-D \gamma u^{\gamma-1}\left(r_{1}\right)=0$ and $u \equiv\left(\frac{D \gamma}{\lambda}\right)^{\frac{1}{2-\gamma}}$ by unique continuation.

The next lemma shows that both integrals in the definition of $I(u)$ and the Lagrange multiplier $\lambda(u)$ in fact only depend on the value $I(u)$. This will be particularly useful in the next section on uniqueness.

Lemma 3.1. Suppose $u$ is a radially decreasing minimizer of $I$. Then

$$
\begin{aligned}
& \frac{1}{2} \int_{\mathbb{R}^{n}}|\nabla u|^{2}=\frac{(2-\gamma) n}{4+(2-\gamma) n} I(u), \quad D \int_{\mathbb{R}^{n}}|u|^{\gamma}=\frac{4}{4+(2-\gamma) n} I(u) \\
& \text { and } \lambda=\lambda(u)=\frac{2(2-\gamma) n+4 \gamma}{4+(2-\gamma) n} I(u)
\end{aligned}
$$

Proof. Let $u_{\mu}(x)=\mu^{n / 2} u(\mu x)$. Clearly, $\left\|u_{\mu}\right\|_{L^{2}}^{2}=\|u\|_{L^{2}}^{2}=1$. Since

$$
I\left(u_{\mu}\right)=\frac{\mu^{2}}{2} \int_{\mathbb{R}^{n}}|\nabla u|^{2}+D \mu^{\frac{(\gamma-2) n}{2}} \int_{\mathbb{R}^{n}}|u|^{\gamma},
$$


it follows from $\left.\partial_{\mu} I\left(u_{\mu}\right)\right|_{\mu=1}=0$ that

$$
\int_{\mathbb{R}^{n}}|\nabla u|^{2}+D \frac{(\gamma-2) n}{2} \int_{\mathbb{R}^{n}}|u|^{\gamma}=0 .
$$

On the other hand,

$$
\frac{1}{2} \int_{\mathbb{R}^{n}}|\nabla u|^{2}+D \int_{\mathbb{R}^{n}}|u|^{\gamma}=I(u) .
$$

Since $\gamma-2<0$, we can solve for $\frac{1}{2} \int_{\mathbb{R}^{n}}|\nabla u|^{2}$ and $D \int_{\mathbb{R}^{n}}|u|^{\gamma}$ in terms of $I(u)$ to obtain the values as claimed.

Noting that $u$ solves the Euler-Lagrange equation (3.1), we can derive another equation by testing (3.1) with $u$ itself to get

$$
\int|\nabla u|^{2}+D \gamma \int|u|^{\gamma}-\lambda=0
$$

and the the identity for $\lambda$ follows by inserting the values found for $\frac{1}{2} \int_{\mathbb{R}^{n}}|\nabla u|^{2}$ and $D \int_{\mathbb{R}^{n}}|u|^{\gamma}$.

We will now prove that decreasing solutions of (3.2) are compactly supported. This is a well-known fact for ground states of (3.2), which holds true under even more general non-linearities (see, e.g., [12]). We will give a short self-contained proof here.

Lemma 3.2. There is no solution $u:(0, \infty) \rightarrow \mathbb{R}$ of

$$
u^{\prime \prime}+\frac{n-1}{r} u^{\prime}+\lambda u-D \gamma|u|^{\gamma-1}=0
$$

with $u>0$ decreasing and $u(r) \rightarrow 0$ as $r \rightarrow \infty$.

Proof. Suppose such a $u$ exists. Since $u(r) \rightarrow 0$ as $r \rightarrow \infty$, there is $r_{0}$ such that $u^{\gamma-2}(r)>\frac{2|\lambda|}{D \gamma}$ and so $D \gamma u^{\gamma-1}(r)-\lambda u(r)>\frac{D \gamma}{2} u^{\gamma-1}(r)$ for all $r \geq r_{0}$. As $u$ decreases, we obtain

$$
u^{\prime \prime}>\frac{D \gamma}{2} u^{\gamma-1} \quad \text { for } r \geq r_{0} .
$$

Note that, in particular, this shows that $u$ is convex on $\left[r_{0}, \infty\right)$. Multiplying this inequality with $u^{\prime} \leq 0$ and integrating over $[s, t]$ with $r_{0} \leq s \leq t$ we obtain

$$
\left(u^{\prime}\right)^{2}(s)-\left(u^{\prime}\right)^{2}(t) \geq D\left(u^{\gamma}(s)-u^{\gamma}(t)\right) .
$$

Since $u(t) \rightarrow 0$ and, by convexity, also $u^{\prime}(t) \rightarrow 0$ as $t \rightarrow \infty$, we then get $u^{\prime}(s) \leq-\sqrt{D} u^{\frac{\gamma}{2}}(s)$, i.e, $u^{-\frac{\gamma}{2}}(s) u^{\prime}(s) \leq$ $-\sqrt{D}$. Integration over $\left[r_{0}, r\right], r>r_{0}$ yields

$$
\frac{2}{2-\gamma}\left(u^{\frac{2-\gamma}{2}}(r)-u^{\frac{2-\gamma}{2}}\left(r_{0}\right)\right) \leq-\sqrt{D}\left(r-r_{0}\right) .
$$

Now letting $r$ tend to $\infty$ gives a contradiction.

An immediate consequence of the strict monotonicity of radially decreasing minimizers and the results in the previous section is that - up to translations and a change of sign - every minimizer is radially decreasing:

Proposition 3.3. Let $u$ be a minimizer of $I$. Then there exists an $a \in \mathbb{R}^{n}$ such that $u=u^{*}(\cdot-a)$ or $u=-u^{*}(\cdot-a)$ a.e.

Proof. By Lemma 2.2, if $u$ is a minimizer, then $u^{*}$ is a minimizer, too, and thus a solution of (3.2). Since $\left(u^{*}\right)^{\prime}<0$ on $\left(0, R=R\left(u^{*}\right)\right)$, again by Lemma 2.2 it follows that $|u|=u^{+}+u^{-}=u^{*}(\cdot-a)$ a.e. for some $a \in \mathbb{R}^{n}$. But then $u=u^{*}(\cdot-a)$ or $u=-u^{*}(\cdot-a)$ a.e. for also $u^{+}+u^{-}(\cdot+4 R)$ is a minimizer, and so $u^{+}+u^{-}(\cdot+4 R)=\left(u^{+}+u^{-}(\cdot+4 R)\right)^{*}(\cdot-b)=u^{*}(\cdot-b)$ for some $b \in \mathbb{R}^{n}$, whence $u^{+}=0$ or $u^{-}=0$ a.e. 
Theorem 1.1(ii) now follows immediately from Lemma 3.2 and Proposition 3.3.

As mentioned, $u$ is smooth on $[0, R)$ and, trivially, on $(R, \infty)$. So for regularity considerations we concentrate on $r=R$. As a first step we examine $u^{\prime}(R)$ :

Lemma 3.4. Let $u$ be a radially decreasing minimizer of $I$. Then $u^{\prime}(R(u))=0$.

(For $\gamma>1$ this follows of course from the Euler-Lagrange equation on $\mathbb{R}^{n}$.)

Proof. Note that $u^{\prime}(R):=\lim _{r} \nearrow_{R} u^{\prime}(r)$ exists in $(-\infty, 0]$ because $u$ is convex near $R$. Set $F(u):=\frac{\lambda}{2} u^{2}-D u^{\gamma}$. Multiplying (3.2) by $r^{n} u^{\prime}$ and integrating from 0 to $s<R$, after integration by parts we find

$$
\begin{aligned}
0= & \frac{s^{n}}{2} u^{\prime}(s)-\frac{n}{2} \int_{0}^{s} r^{n-1}\left(u^{\prime}\right)^{2} \mathrm{~d} r+(n-1) \int_{0}^{s} r^{n-1}\left(u^{\prime}\right)^{2} \mathrm{~d} r \\
& +s^{n}\left(\frac{\lambda}{2} u^{2}(s)-D u^{\gamma}(s)\right)-n \int_{0}^{s} r^{n-1}\left(\frac{\lambda}{2} u^{2}-D u^{\gamma}\right) \mathrm{d} r .
\end{aligned}
$$

Sending $s \rightarrow R$ and changing to $n$-dimensional integrals gives

$$
\begin{aligned}
\frac{n \omega_{n} R^{n}}{2} u^{\prime}(R) & =-\frac{n-2}{2} \int_{B_{R}}|\nabla u|^{2} \mathrm{~d} x+n \int_{B_{R}} \frac{\lambda}{2} u^{2}-D u^{\gamma} \mathrm{d} x \\
& =\frac{-(n-2)(2-\gamma) n+((2-\gamma) n+2 \gamma) n-4 n}{4+(2-\gamma) n} I(u)=0
\end{aligned}
$$

by Lemma 3.1.

Proposition 3.5. Let $u$ be a radially decreasing minimizer of $I, \beta:=\frac{2}{2-\gamma}$. Then, for all $k \in \mathbb{N}, \frac{u^{(k)}}{(R-r)^{\beta-k}} \in$ $C([0, R])$. In particular, $u \in C^{1}$ for all $\gamma$ and

$$
\begin{aligned}
& \lim _{r \nearrow R} \frac{u(r)}{(R-r)^{\frac{2}{2-\gamma}}}=\left(\frac{\sqrt{D}(2-\gamma)}{\sqrt{2}}\right)^{\frac{2}{2-\gamma}} \text { and } \\
& \lim _{r \nearrow R} \frac{u^{\prime}(r)}{(R-r)^{\frac{\gamma}{2-\gamma}}}=-\sqrt{2 D}\left(\frac{\sqrt{D}(2-\gamma)}{\sqrt{2}}\right)^{\frac{\gamma}{2-\gamma}} .
\end{aligned}
$$

Note that this shows that $u \in W^{\lfloor\beta\rfloor+1, p}\left(\mathbb{R}^{n}\right)$ for all $p<\frac{1}{[\beta\rfloor+1-\beta}$ if $\beta \notin \mathbb{N}, u \in W^{\beta, \infty}\left(\mathbb{R}^{n}\right)$ if $\beta \in \mathbb{N}$. As a consequence, $u \in W^{2,1}\left(\mathbb{R}^{n}\right)$ is a distributional solution of $\Delta u+\lambda u-D \gamma u^{\gamma-1}=0$ on all of $\mathbb{R}^{n}$ for every $\gamma \in(0,2)$. Proof. Since by Lemma 3.4, monotone convergence and by (3.2)

$$
\frac{1}{2}\left(u^{\prime}\right)^{2}(r)=-\int_{r}^{R} u^{\prime \prime} u^{\prime} \mathrm{d} t=\int_{r}^{R} \frac{n-1}{t}\left(u^{\prime}\right)^{2} \mathrm{~d} t-\frac{\lambda}{2} u^{2}(r)+D u^{\gamma}(r),
$$

and since $\int_{r}^{R} \frac{n-1}{t}\left(u^{\prime}\right)^{2} \mathrm{~d} t \leq(R-r) \frac{n-1}{r}\left(u^{\prime}\right)^{2}(r) \ll\left(u^{\prime}\right)^{2}(r)$ and $u^{2}(r) \ll u^{\gamma}(r)$ as $r \rightarrow R$, it follows that $\lim _{r} \nearrow_{R} u^{-\gamma}(r)\left(u^{\prime}\right)^{2}(r)=2 D$ and thus

$$
\lim _{r \nearrow R} u^{-\frac{\gamma}{2}}(r) u^{\prime}(r)=-\sqrt{2 D}
$$

Now L'Hospital's rule implies that

$$
\lim _{r \nearrow R} \frac{u^{\frac{2-\gamma}{2}}(r)}{R-r}=\lim _{r \nearrow R}-\frac{2-\gamma}{2} u^{-\frac{\gamma}{2}}(r) u^{\prime}(r)=\frac{2-\gamma}{2} \sqrt{2 D} .
$$

This, together with (3.4), yields the asymptotic behavior of $u$ and $u^{\prime}$. 
Now note that for each $k \in \mathbb{N}$ there are polynomials $\varphi_{k, i}$ in $\frac{1}{r}$ and numbers $a_{k, i}, b_{k, i}, i \in\left\{1, \ldots, m_{k}\right\}$, such that

$$
u^{(k)}=\sum_{i=1}^{m_{k}} \varphi_{k, i} u^{a_{k, i}}\left(u^{\prime}\right)^{b_{k, i}}
$$

with $\beta a_{k, i}+(\beta-1) b_{k, i} \geq \beta-k$, where $\beta:=\frac{2}{2-\gamma}$. (This clear for $k=0,1$ and follows easily by induction for general $k$ from (3.2).) The claim now follows from the asymptotic behavior of $u$ and $u^{\prime}$ near $r=R$.

\section{UNIQUENESS OF MINIMIZERS}

We will now prove that minimizers are (up to translations and a change of sign) unique. By Proposition 3.3 it suffices to prove uniqueness in the class of radially symmetric and decreasing functions. We split the proof into several lemmas. Note that Lemma 3.1 has two important consequences. Firstly, a variational argument implies that the difference of two minimizers has to change sign at least twice (see Lem. 4.1). Secondly, we may study the common Euler-Lagrange equation to show by PDE-methods (mainly Green's formula) that the difference of two minimizers cannot change sign more than once, see Proposition 4.2. (In what follows we will repeatedly use that, if $u_{1}, u_{2}$ are two different solutions of $(3.2)$, then $u_{1}(r)=u_{2}(r)$ implies $u_{1}^{\prime}(r) \neq u_{2}^{\prime}(r)$ for each $r \in\left(0, \min \left\{R\left(u_{1}\right), R\left(u_{2}\right)\right\}\right)$ by unique continuation. Also we will view radially symmetric functions in $n$ variables $x=\left(x_{1}, \ldots, x_{n}\right)$ as functions of the radial variable $r=|x| \geq 0$ and vice versa whenever convenient.)

The case $n=1$, which can be solved explicitly, will be treated separately in the next section. So assume for now that $n \geq 2$.

Lemma 4.1. Suppose $u_{1}$ and $u_{2}$ are radially decreasing minimizers of $I, u_{1} \not \equiv u_{2}$. Then $u_{1}-u_{2}$ has to change sign at least twice, i.e., $u_{1}-u_{2}$ has at least two zeros in $(0, R), R:=\min \left\{R\left(u_{1}\right), R\left(u_{2}\right)\right\}$.

Proof. $u_{1}-u_{2}$ has to change sign at least once, for otherwise $\int_{\mathbb{R}^{n}}\left|u_{1}\right|^{2}$ and $\int_{\mathbb{R}^{n}}\left|u_{2}\right|^{2}$ cannot both be equal to 1 .

Suppose $u_{1}-u_{2}$ changes sign only once, without loss of generality say $u_{1}(r)>u_{2}(r)$ on $\left(0, r_{1}\right)$ and $u_{1}(r)<$ $u_{2}(r)$ on $\left(r_{1}, R_{2}\right), R_{2}=R\left(u_{2}\right)$. Let $\varphi:=u_{1}^{2}-u_{2}^{2}$. By strict concavity of $(0, \infty) \ni t \mapsto t^{\frac{\gamma}{2}}$ and since $\left(0, R_{2}\right) \ni$ $r \mapsto u_{2}^{\gamma-2}(r)$ is strictly increasing, we find

$$
u_{1}^{\gamma}(r)=\left(u_{2}^{2}(r)+\varphi(r)\right)^{\frac{\gamma}{2}} \leq u_{2}^{\gamma}(r)+\frac{\gamma}{2} u_{2}^{\gamma-2}(r) \varphi(r) \leq u_{2}^{\gamma}(r)+\frac{\gamma}{2} u_{2}^{\gamma-2}\left(r_{1}\right) \varphi(r),
$$

where the inequalities are strict for $r \neq r_{1}$. Integrating over the ball of radius $R_{2}$ centered at the origin we arrive at $\int_{\mathbb{R}^{n}}\left|u_{1}\right|^{\gamma}<\int_{\mathbb{R}^{n}}\left|u_{2}\right|^{\gamma}$ since $\int_{\mathbb{R}^{n}} \varphi=0$. But this contradicts the assertion of Lemma 3.1.

For the sake of notational convenience we note that, by the results of the previous section, for every symmetrically decreasing minimizer $u$ of $I$ the function $\left(\frac{D \gamma}{\lambda}\right)^{-\frac{1}{2-\gamma}} u\left(\frac{\cdot}{\sqrt{\lambda}}\right)$ (with $\lambda$ as in Lem. 3.1) is a decreasing classical solution of

$$
\Delta u+f(u)=0 \text { on }(0, R), \quad u(R)=0
$$

with $f(u)=u-u^{\alpha}, \alpha:=\gamma-1$ and $R=R(u)$ such that $u>0$ on $[0, R)$ and $u^{\prime}$ is bounded. In case $\gamma>1$ it even solves

$$
\Delta u+f(u)=0 \text { on }(0, \infty), \quad u=0 \text { on }[R, \infty)
$$

Proposition 4.2. Let $u_{1} \in C^{2}\left(\left[0, R\left(u_{1}\right)\right)\right), u_{2} \in C^{2}\left(\left[0, R\left(u_{2}\right)\right)\right)$ be two different functions such that

(i) $-1<\alpha \leq 0$ and $u_{1}, u_{2}$ are solutions of (4.1), or

(ii) $0<\alpha<1$ and $u_{1}, u_{2}$ are solutions of (4.2),

then their graphs cannot intersect twice above 0 . 
We split the proof into several lemmas.

Lemma 4.3. Suppose $u_{1}, u_{2}$ are decreasing solutions solutions of (4.1) with $u_{1} \not \equiv u_{2}$. Then the graphs of $u_{1}$ and $u_{2}$ cannot intersect twice in $[0, \infty) \times(0,1]$.

Proof. Suppose there were $r_{1}<r_{2}$ such that $1 \geq u_{1}\left(r_{1}\right)=u_{2}\left(r_{1}\right)>u_{1}\left(r_{2}\right)=u_{2}\left(r_{2}\right)>0$. Without loss of generality assume $u_{2}>u_{1}$ on $\left(r_{1}, r_{2}\right)$. Since $u_{1}$ and $u_{2}$ are strictly decreasing on $\left[r_{1}, r_{2}\right]$, their respective inverses $v_{1}, v_{2}:\left[u_{1}\left(r_{2}\right), u_{1}\left(r_{1}\right)\right] \rightarrow\left[r_{1}, r_{2}\right]$ exist. Choose $\bar{u} \in\left[u_{1}\left(r_{2}\right), u_{1}\left(r_{1}\right)\right]$ such that $\frac{v_{2}(u)}{v_{1}(u)}$ is maximal at $u=\bar{u}$ with value, say, $\mu>1$. Then $v_{2} \leq \mu v_{1}$ with equality at $\bar{u}$ and strict inequality at $u_{1}\left(r_{1}\right)$ and $u_{1}\left(r_{2}\right)$.

By $u^{(\mu)}$ denote the inverse function of $\mu v_{1}:\left[u_{1}\left(r_{1}\right), u_{1}\left(r_{2}\right)\right] \rightarrow\left[\mu r_{1}, \mu r_{2}\right]$, i.e., $u^{(\mu)}(r)=u_{1}\left(\frac{r}{\mu}\right)$. Then $v_{2}(\bar{u}) \in\left(\mu r_{1}, r_{2}\right)$ and $u^{(\mu)} \geq u_{2}$ on $\left[\mu r_{1}, r_{2}\right]$ with equality at $r=v_{2}(\bar{u})$ and strict inequality on $\left\{\mu r_{1}, r_{2}\right\}$, whence we can also find an $\eta>0$ such that $\mu\left(r_{1}+\eta\right)<v_{2}(\bar{u})<r_{2}$ and $u^{(\mu)}>u_{2}$ on $\left\{\mu\left(r_{1}+\eta\right), r_{2}\right\}$. Then for any $\delta>0$ we may choose an open interval $V_{\delta} \subset\left(\mu\left(r_{1}+\eta\right), r_{2}\right)$ containing $v_{2}(\bar{u})$ such that $0 \leq u^{(\mu)}-u_{2}<\delta$ on $V_{\delta}$ and $u^{(\mu)}-u_{2}>0$ on $\partial V_{\delta}$.

This finally leads to a contradiction if $\delta$ is chosen so small that $f\left(u_{2}\right)-f\left(u^{(\mu)}\right)<a:=\left(1-\frac{1}{\mu^{2}}\right) \min \left\{-f\left(u_{1}\left(r_{1}+\right.\right.\right.$ $\left.\eta)),-f\left(u_{1}\left(\frac{r_{2}}{\mu}\right)\right)\right\}$ on $V_{\delta}$, for then

$$
\begin{aligned}
\Delta\left(u^{(\mu)}-u_{2}\right)(r) & =\frac{1}{\mu^{2}}\left(\Delta u_{1}\right)\left(\frac{r}{\mu}\right)-\Delta u_{2}(r)=f\left(u_{2}(r)\right)-\frac{1}{\mu^{2}} f\left(u^{(\mu)}(r)\right) \\
& <\left(1-\frac{1}{\mu^{2}}\right) f\left(u^{(\mu)}(r)\right)+a \leq 0
\end{aligned}
$$

since $f\left(u^{(\mu)}\right) \leq \max \left\{f\left(u_{1}\left(r_{1}+\eta\right)\right), f\left(u_{1}\left(\frac{r_{2}}{\mu}\right)\right)\right\}$ on $\left[\mu\left(r_{1}+\eta\right), r_{2}\right]$ (by convexity of $f$ if $\alpha \in(0,1)$ and by monotonicity of $f$ if $\alpha \in(-1,0])$. This, however, cannot be true because $u^{(\mu)}-u_{2}$ does not attain its minimum over $V_{\delta}$ on the boundary $\partial V_{\delta}$.

Proof of Proposition 4.2(i). Suppose the graphs of $u_{1}$ and $u_{2}$ intersect twice above 0. By Lemma 4.3 there exists $r_{1}$ such that $u_{1}\left(r_{1}\right)=u_{2}\left(r_{1}\right)>1$. Without loss of generality we may assume that $u_{1}>u_{2}$ on $\left(r_{1}-\varepsilon, r_{1}\right)$ for $\varepsilon>0$ sufficiently small. Let $r_{0}=\inf \left\{r>0: u_{1}>u_{2}\right.$ on $\left.\left(r, r_{1}\right)\right\}$. Since $u_{1}^{\prime}\left(r_{1}\right)<u_{2}^{\prime}\left(r_{1}\right), u_{1}\left(r_{1}\right)=u_{2}\left(r_{1}\right)>1$ and either $r_{0}=0$ (in which case the first term on the right hand side of the following formula vanishes) or else $r_{0}>0$ and hence $u_{1}\left(r_{0}\right)=u_{2}\left(r_{0}\right)>u_{2}\left(r_{1}\right)>1$ with $u_{1}^{\prime}\left(r_{0}\right)>u_{2}^{\prime}\left(r_{0}\right)$, we obtain for $v_{i}=u_{i}-1$

$$
0>r_{0}^{n-1}\left(-v_{2}\left(r_{0}\right) v_{1}^{\prime}\left(r_{0}\right)+v_{1}\left(r_{0}\right) v_{2}^{\prime}\left(r_{0}\right)\right)+r_{1}^{n-1}\left(v_{2}\left(r_{1}\right) v_{1}^{\prime}\left(r_{1}\right)-v_{1}\left(r_{1}\right) v_{2}^{\prime}\left(r_{1}\right)\right),
$$

which by Green's formula implies

$$
0>\int_{r_{0}}^{r_{1}} r^{n-1}\left(v_{2} \Delta v_{1}-v_{1} \Delta v_{2}\right) \mathrm{d} r=\int_{r_{0}}^{r_{1}} r^{n-1} v_{1} v_{2}\left(\frac{f\left(v_{2}+1\right)}{v_{2}}-\frac{f\left(v_{1}+1\right)}{v_{1}}\right) \mathrm{d} r .
$$

But this cannot be true as $v_{1}>v_{2}>0$ on $\left(r_{0}, r_{1}\right)$ and the function $v \mapsto \frac{f(v+1)}{v}$ is decreasing on $(0, \infty)$ : its derivative is given by

$$
v \mapsto v^{-2}\left((v+1)^{\alpha}+\alpha(v+1)^{\alpha-1}(-v)-1\right)
$$

which is non-positive by the convexity of $v \mapsto v^{\alpha}$ for $\alpha \leq 0$.

We now turn to the case $\gamma>1$. Here we will need the following monotonicity lemma from [5] (going back to [10]). We will include the proof - following [6] - for the sake of completeness.

Lemma 4.4. Let $R>0$ and suppose $u \in C^{2}([0, R))$ is a decreasing solution of (4.1). Then $\frac{r u^{\prime}}{u}$ is strictly decreasing on $(0, R)$. 
Proof. On $(0, R)$ we have

$$
-r^{n-1} u^{2}\left(\frac{r u^{\prime}}{u}\right)^{\prime}=r^{n-1}\left(\left(r u^{\prime}\right) u^{\prime}-\left(r u^{\prime}\right)^{\prime} u\right)
$$

Since $\Delta\left(r u^{\prime}\right)=-r u^{\prime} f^{\prime}(u)-2 f(u)$, Green's formula yields

$$
\begin{aligned}
r^{n-1}\left(\left(r u^{\prime}\right) u^{\prime}-\left(r u^{\prime}\right)^{\prime} u\right) & =\int_{0}^{r}\left(t u^{\prime} \Delta u-u \Delta\left(t u^{\prime}\right)\right) t^{n-1} \mathrm{~d} t \\
& =\int_{0}^{r}\left(-u^{\prime} f(u)+u u^{\prime} f^{\prime}(u)\right) t^{n} \mathrm{~d} t+\int_{0}^{r} 2 u f(u) t^{n-1} \mathrm{~d} t \\
& =\int_{0}^{r}(1-\alpha) u^{\alpha} u^{\prime} t^{n} \mathrm{~d} t+\int_{0}^{r} 2 u f(u) t^{n-1} \mathrm{~d} t \\
& =\frac{1-\alpha}{1+\alpha} u^{1+\alpha}(r) r^{n}+\int_{0}^{r}\left(2 u^{2}-\left(2+\frac{(1-\alpha) n}{1+\alpha}\right) u^{1+\alpha}\right) t^{n-1} \mathrm{~d} t
\end{aligned}
$$

for $r<R$. Now note $(0, \infty) \ni u \mapsto 2 u^{2}-\left(2+\frac{(1-\alpha) n}{1+\alpha}\right) u^{1+\alpha}$ has a unique zero $\bar{u}$, say. Then if $u(r) \geq \bar{u}$, the last integral in (4.4) is non-negative. If on the other hand $u(r)<\bar{u}$, then this integral can be bounded from below by the integral up to $R$ as the integrand is negative on $(r, R)$ :

$$
\int_{0}^{r}\left(2 u^{2}-\left(2+\frac{(1-\alpha) n}{1+\alpha}\right) u^{1+\alpha}\right) t^{n-1} \mathrm{~d} t \geq \int_{0}^{R}\left(2 u^{2}-\left(2+\frac{(1-\alpha) n}{1+\alpha}\right) u^{1+\alpha}\right) t^{n-1} \mathrm{~d} t=0,
$$

where the last equality followed from (4.4) with $r$ sent to $R$ (note that $\left(r u^{\prime}\right)^{\prime} u=(2-n) u^{\prime} u-r u f(u)$ ), or alternatively from an explicit evaluation with the help of Lemma 3.1. So also in this case the last integral in (4.4) is non-negative. Since $u^{1+\alpha}>0$ on $(0, R)$ the claim then follows from (4.3) and (4.4).

Proof of Proposition 4.2(ii). Suppose $u_{1}$ and $u_{2}$ intersect at least twice above 0 . Then by Lemma 4.3 we may assume that there exist $0<r_{1}<r_{2}$ such that $u_{1}\left(r_{1}\right)=u_{2}\left(r_{1}\right)>1, u_{1}\left(r_{2}\right)=u_{2}\left(r_{2}\right)>0$ and $u_{2}>u_{1}$ on $\left(r_{1}, r_{2}\right)$. Let $r_{0}:=\inf \left\{r>0: u_{1}>u_{2}\right.$ on $\left.\left(r, r_{1}\right)\right\}$ and $r_{3}:=\sup \left\{r>r_{2}: u_{1}>u_{2}\right.$ on $\left.\left(r_{2}, r\right)\right\}$. Set $w:=u_{1}-u_{2}$ and note that, by convexity of $f$,

$$
\Delta w+f^{\prime}\left(u_{1}\right) w=f\left(u_{2}\right)-f\left(u_{1}\right)-f^{\prime}\left(u_{1}\right)\left(u_{2}-u_{1}\right) \geq 0
$$

on $\left(0, R_{1}\right), R_{1}=R\left(u_{1}\right)$.

Let $v:=r u_{1}^{\prime}+\beta u_{1}$ and $\beta:=\frac{2\left(u_{1}^{1-\alpha}\left(r_{1}\right)-1\right)}{1-\alpha}>0$. An elementary calculation shows that

$$
\Delta v+f^{\prime}\left(u_{1}\right) v=\Phi\left(u_{1}\right):=u_{1}^{\alpha}\left((1-\alpha) \beta-2\left(u_{1}^{1-\alpha}-1\right)\right)
$$

in particular that $\Phi\left(u_{1}\right)<0$ on $\left(0, r_{1}\right)$ and $\Phi\left(u_{1}\right)>0$ on $\left(r_{1}, R_{1}\right)$. Note also that $v<0$ on $\left(r_{1}, R_{1}\right)$ : if this were not the case, then $v>0$ on $\left(0, r_{1}\right)$ since by Lemma $4.4 v$ can change sign at most once on $\left(0, R_{1}\right)$. But then using (4.5) and (4.6) we would find

$$
v \Delta w-w \Delta v>0 \text { on }\left(r_{0}, r_{1}\right)
$$

and, by Green's formula, obtain that

$$
-r_{0}^{n-1} v\left(r_{0}\right) w^{\prime}\left(r_{0}\right)+r_{1}^{n-1} v\left(r_{1}\right) w^{\prime}\left(r_{1}\right)+r_{0}^{n-1} w\left(r_{0}\right) v^{\prime}\left(r_{0}\right)-r_{1}^{n-1} w\left(r_{1}\right) v^{\prime}\left(r_{1}\right)>0 .
$$

Since $v\left(r_{0}\right), v\left(r_{1}\right) \geq 0, w^{\prime}\left(r_{1}\right)<0 \leq r_{0}^{n-1} w^{\prime}\left(r_{0}\right)$ and $r_{0}^{n-1} w\left(r_{0}\right)=r_{1}^{n-1} w\left(r_{1}\right)=0$, this leads to a contradiction. 
As a consequence we get from (4.5) and (4.6) that

$$
w \Delta v-v \Delta w>0 \text { on }\left(r_{2}, r_{3}\right)
$$

Again by Green's formula we obtain

$$
r_{2}^{n-1} v\left(r_{2}\right) w^{\prime}\left(r_{2}\right)-r_{3}^{n-1} v\left(r_{3}\right) w^{\prime}\left(r_{3}\right)>0 .
$$

But this contradicts $v\left(r_{2}\right)<0, w^{\prime}\left(r_{2}\right)>0, v\left(r_{3}\right) \leq 0, w^{\prime}\left(r_{3}\right) \leq 0$.

Theorem 1.1(iii) now immediately follows from Lemmas 4.1, 3.1 and Proposition 4.2.

\section{Explicitly solvable CASES}

In this last section we examine special cases where the minimizers can be determined explicitly. In particular, this is possible in one dimension (as has been noted before by Biskup and König, unpublished notes) and when the Euler-Lagrange equation is linear. Interestingly, it turns out that also the limit for $\gamma$ close to the critical exponent 2 yields an explicit asymptotic expression for the minimizers.

\subsection{The one-dimensional case}

Proposition 5.1. Let $n=1$. There exists a unique radially decreasing minimizer $u$ of $I$, which is given by

$$
u(r)= \begin{cases}\left(\frac{2 D}{\lambda}\right)^{\frac{1}{2-\gamma}} \cos ^{\frac{2}{2-\gamma}\left(\frac{\sqrt{\lambda}(2-\gamma) r}{2}\right)} & \text { for } 0 \leq r \leq \frac{\pi}{\sqrt{\lambda}(2-\gamma)} \\ 0 & \text { for } r \geq \frac{\pi}{\sqrt{\lambda}(2-\gamma)}\end{cases}
$$

where $\lambda=(2 D)^{\frac{4}{6-\gamma}}\left(\frac{\sqrt{\pi} \Gamma\left(\frac{6-\gamma}{4-2 \gamma}\right)}{\Gamma\left(\frac{2}{2-\gamma}\right)}\right)^{\frac{4-2 \gamma}{6-\gamma}}$. Furthermore, $I(u)=\frac{6-\gamma}{4+2 \gamma} \lambda$.

Proof. Suppose $u$ is a radially decreasing minimizer of $I$ in one dimension, so that

$$
u^{\prime \prime}+\lambda u-D \gamma u^{\gamma-1}=0, \quad u^{\prime}(0)=0, \quad u(R(u))=u^{\prime}(R(u))=0
$$

by (3.2) and Lemmas 3.2 and 3.4. This equation can be solved explicitly, e.g., by first multiplying with $2 u^{\prime}$ and integrating over $(r, R)$ to obtain

$$
u^{\prime}(r)=-\sqrt{-\lambda u^{2}(r)+2 D u^{\gamma}(r)}
$$

This is a first order autonomous equation and it easily follows that the unique solution of (5.2) that strictly decreases on $(0, R(u))$ is given by (5.1). The value of $\lambda$ follows from

$$
1=\|u\|_{L^{2}}^{2}=2\left(\frac{2 D}{\lambda}\right)^{\frac{2}{2-\gamma}} \int_{0}^{\frac{\pi}{\sqrt{\lambda}(2-\gamma)}} \cos ^{\frac{4}{2-\gamma}}\left(\frac{\sqrt{\lambda}(2-\gamma)}{2} r\right) \mathrm{d} r=(2 D)^{\frac{2}{2-\gamma}} \frac{\sqrt{\pi} \Gamma\left(\frac{6-\gamma}{4-2 \gamma}\right)}{\Gamma\left(\frac{2}{2-\gamma}\right)} \lambda^{-\frac{6-\gamma}{4-2 \gamma}}
$$

and Lemma 3.1 implies $I(u)=\frac{6-\gamma}{4+2 \gamma} \lambda$.

\subsection{Linear Euler-Lagrange equations}

For the sake of completeness we also briefly discuss $I_{\gamma}$ for the special values $\gamma=0,1$ that lead to explicitly solvable Euler-Lagrange equations. Here, for $\gamma=0$, the functional $I_{\gamma}$ is interpreted as

$$
I_{0}(u)=\frac{1}{2} \int_{\mathbb{R}^{n}}|\nabla u(x)|^{2} \mathrm{~d} x+D \int_{\mathbb{R}^{n}} g(u) \mathrm{d} x
$$


with $g(u)=1$ if $u \neq 0$ and $g(0)=0$. (By the classical Rayleigh-Faber-Krahn inequality, the minimizers of this functional are of course well known.)

Let $U(r)=r^{\frac{2-n}{2}} J_{\frac{n-2}{2}}(r), J_{\frac{n-2}{2}}$ the Bessel function of the first kind with parameter $\frac{n-2}{2}$, so that $x \mapsto U(|x|)$ is an eigenfunction of the Laplacian with eigenvalue 1.

Proposition 5.2. The minimizers of $I_{0}$, respectively $I_{1}$, are - up to translations or a change of sign - given by

$$
u_{0}(x)= \begin{cases}\mu_{0} U\left(\sqrt{\lambda_{0}}|x|\right) & \text { for }|x| \leq \frac{r_{0}}{\sqrt{\lambda_{0}}} \\ 0 & \text { for }|x|>\frac{r_{0}}{\sqrt{\lambda_{0}}}\end{cases}
$$

where $r_{0}$ is the first zero of $U$, respectively

$$
u_{1}(x)= \begin{cases}\frac{D \gamma}{\lambda_{1}}\left(\frac{U\left(\sqrt{\lambda_{1}}|x|\right)}{\left|U\left(r_{1}\right)\right|}+1\right) & \text { for }|x| \leq \frac{r_{1}}{\sqrt{\lambda_{1}}} \\ 0 & \text { for }|x|>\frac{r_{1}}{\sqrt{\lambda_{1}}}\end{cases}
$$

where $r_{1}$ is the point at which $U$ assumes its first minimum, for suitable $\mu_{0}, \lambda_{0}, \lambda_{1}>0$. The values of $\mu_{0}$, $\lambda_{0}$ and $\lambda_{1}$ can be computed explicitly.

Proof. As in the previous sections we see that minimizers of $I_{0}$ are radially decreasing up to translations or a change of sign and we easily infer from $\Delta u_{0}+\lambda_{0} u_{0}=0$ on $B_{R}$ that $u_{0}$ is of the form (5.3). The condition $\left\|u_{0}\right\|_{L^{2}}=1$ leads to $\mu_{0}=\|U\|_{L^{2}\left(\mathbb{R}^{n}\right)}^{-1} \lambda_{0}^{\frac{n}{4}}$. But then

$$
I_{0}(u)=\frac{1}{2}\|\nabla U\|_{L^{2}\left(\mathbb{R}^{n}\right)}^{2}\|U\|_{L^{2}\left(\mathbb{R}^{n}\right)}^{-2} \lambda_{0}+\|U\|_{L^{2}\left(\mathbb{R}^{n}\right)}^{-\gamma} D \int_{B_{r_{1}}} U^{\gamma} \mathrm{d} x \lambda_{0}^{\frac{(\gamma-2) n}{4}}
$$

and minimizing with respect to $\lambda_{0}$ concludes the proof for $\gamma=0$.

If $\gamma=1$, noting that $\Delta\left(u_{1}-\frac{D \gamma}{\lambda_{1}}\right)=\lambda_{1}\left(u_{1}-\frac{D \gamma}{\lambda_{1}}\right)$, we readily infer that $u_{1}$ is indeed of the form (5.4). $\lambda_{1}$ can then then be determined from $\left\|u_{1}\right\|_{L^{2}}=1$.

\subsection{Behavior near the critical exponent}

We now investigate the behavior of the minimizers of $I=I_{\gamma}$ when $\gamma$ is close to 2 . Note that inf ${ }_{\mathcal{A}} I_{2}=D$, but the minimum is not attained. In fact, as $\gamma$ approaches 2 , the minimizers of $I_{\gamma}$ are more and more smeared out over $\mathbb{R}^{n}$, and so we introduce the rescaling $w(x)=(2-\gamma)^{-\frac{n}{4}} u\left(\frac{x}{\sqrt{2-\gamma}}\right)$. Alternatively, one can rescale $D$ in order to obtain a non-trivial limit of $I_{\gamma, \frac{D}{2-\gamma}}$. In [9] König gives a formal argument that in this regime the minimizers may be expected to approach a Gaussian function. We will make this precise by proving the following result:

Theorem 5.3. Let $u_{\gamma, D}$ be the unique radially decreasing minimizer of $I_{\gamma, D}$. If $w_{\gamma, D}$ are the rescaled functions $w_{\gamma, D}=(2-\gamma)^{-\frac{n}{4}} u_{\gamma, D}\left(\frac{\cdot}{\sqrt{2-\gamma}}\right)$, then as $\gamma \rightarrow 2$,

$$
\lim _{\gamma \nearrow 2} w_{\gamma, D}=\lim _{\gamma \nearrow 2} u_{\gamma, \frac{D}{2-\gamma}}=w, \quad \text { where } \quad w(x)=\left(\frac{D}{\pi}\right)^{\frac{n}{2}} \mathrm{e}^{-D|x|^{2}}
$$

strongly in $H^{1}$ and

$$
\begin{aligned}
\lim _{\gamma / 2} \frac{I_{\gamma, D}\left(u_{\gamma, D}\right)-D(2-\gamma)^{-\frac{(2-\gamma) n}{4}}}{2-\gamma} & =\lim _{\gamma / 2} I_{\gamma, \frac{D}{2-\gamma}}\left(u_{\gamma, \frac{D}{2-\gamma}}\right)-\frac{D}{2-\gamma} \\
& =\frac{D n}{2}\left(1+\frac{\log \pi-\log D}{2}\right)
\end{aligned}
$$


As in Corollary 2.6 we will use a $\Gamma$-convergence argument to prove this by considering the functionals

$$
J_{\gamma}(w):=\frac{1}{2} \int_{\mathbb{R}^{n}}|\nabla w|^{2}+D c_{\gamma} \int_{\mathbb{R}^{n}} \frac{|w|^{\gamma}-|w|^{2}}{2-\gamma}, \quad c_{\gamma}:=(2-\gamma)^{-\frac{(2-\gamma) n}{4}},
$$

on $\mathcal{A}$. It is not hard to see that $u_{\gamma}$ is a minimizer of $I_{\gamma}$ if and only if its rescaling $w_{\gamma}=(2-\gamma)^{-\frac{n}{4}} u\left(\frac{\text {. }}{\sqrt{2-\gamma}}\right)$ is a minimizer of $J_{\gamma}$.

Before we state the relevant result, we recall the logarithmic Sobolev inequality (see, e.g., [11]): For any $f \in H^{1}\left(\mathbb{R}^{n}\right)$ with $\|f\|_{L^{2}}=1$ and any number $a>0$ it holds

$$
a^{2} \int_{\mathbb{R}^{n}}|\nabla f|^{2}-\int_{\mathbb{R}^{n}}|f|^{2} \log \left(|f|^{2}\right) \geq n\left(1+\frac{\log \pi}{2}+\log a\right)
$$

with equality if and only if $f(x)= \pm\left(2 \pi a^{2}\right)^{-\frac{n}{2}} \mathrm{e}^{-\frac{x^{2}}{2 a^{2}}}$ after a suitable translation of $f$.

Proposition 5.4. The functionals $J_{\gamma} \Gamma$-converge to the functional

$$
J(w):=\frac{1}{2} \int_{\mathbb{R}^{n}}|\nabla w|^{2}-D \int_{\mathbb{R}^{n}}|w|^{2} \log |w|
$$

on $\mathcal{A}$ as $\gamma \rightarrow 2$ with respect to the strong $L^{2}$ - and the weak $H^{1}$-topology. Moreover, every recovery sequence converges strongly in $H^{1}$.

Proof. Note first that clearly $c_{\gamma} \rightarrow 1$ as $2 \rightarrow \gamma$ and that by Taylor-expansion we have

$$
\frac{|w|^{\gamma}-|w|^{2}}{2-\gamma}=-|w|^{\xi} \log |w|=-|w|^{2} \log |w|+\frac{2-\gamma}{2}|w|^{\zeta}(\log |w|)^{2}
$$

for some $\xi=\xi(|w|), \zeta=\zeta(|w|) \in(\gamma, 2)$.

To construct recovery sequences $\left(w_{\gamma}\right)$ we assume first that $w \in \mathcal{A}$ has compact support. Then indeed $w_{\gamma}:=w$ for all $\gamma$ satisfies

$$
J_{\gamma}\left(w_{\gamma}\right)-J(w)=\left(c_{\gamma}-1\right) D \int_{\mathbb{R}^{n}} \frac{|w|^{\gamma}-|w|^{2}}{2-\gamma}+D \frac{2-\gamma}{2} \int_{\mathbb{R}^{n}}|w|^{\zeta}(\log |w|)^{2}
$$

Since $\left|\frac{|w|^{\gamma}-|w|^{2}}{2-\gamma}\right|=|w|^{\xi}|\log | w|| \leq C\left(|w|+|w|^{2^{*}}\right)$ and $|w|^{\zeta}(\log |w|)^{2} \mid \leq C\left(|w|+|w|^{2^{*}}\right)$ we obtain from Sobolev's embedding theorem that both of the integrals in (5.7) are bounded by $C\|w\|_{H^{1}}$, whence $J_{\gamma}\left(w_{\gamma}\right)-J(w) \rightarrow 0$ as $\gamma \rightarrow 2$.

The existence of a recovery sequence for general $w \in \mathcal{A}$ follows from a general argument in the theory of $\Gamma$-convergence if one notices that $w$ can be approximated by $w_{R} \in \mathcal{A}$ with compact support such that $J\left(w_{R}\right) \rightarrow J(w)$ as $R \rightarrow \infty$ : Choose $v_{R}$, supported on $B_{R}$, with $\left|v_{R}\right| \nearrow|w|$ a.e. and $v_{R} \rightarrow w$ in $H^{1}$ as $R \rightarrow \infty$ and set $w_{R}=\frac{v_{R}}{\left\|v_{R}\right\|_{L^{2}}}$ (cf. the proof of Prop. 2.4). Then $\int\left|\nabla w_{R}\right|^{2} \rightarrow \int|\nabla w|^{2}$ and

$$
\begin{aligned}
\int_{\mathbb{R}^{n}}\left|w_{R}\right|^{2} \log \left|w_{R}\right|=- & \log \left\|v_{R}\right\|_{L^{2}}+\frac{1}{\left\|v_{R}\right\|_{L^{2}}^{2}} \int_{\left\{|w| \leq \frac{1}{\sqrt{e}}\right\}}\left|v_{R}\right|^{2} \log \left|v_{R}\right| \\
& +\frac{1}{\left\|v_{R}\right\|_{L^{2}}^{2}} \int_{\left\{|w|>\frac{1}{\sqrt{e}}\right\}}\left|v_{R}\right|^{2} \log \left|v_{R}\right| .
\end{aligned}
$$

By monotone convergence, the first integral converges to $\int_{\left\{|w| \leq \frac{1}{\sqrt{e}}\right\}} w^{2} \log |w|$ as $R \rightarrow \infty$. For the second integral note that $|v|_{R}$ being bounded in $H^{1}$, it is also bounded in $L^{2^{*}}$ and thus $\left|v_{R}^{2} \log \right| v_{R}||$ equiintegrable 
on the set $\left\{|w|>\frac{1}{\sqrt{e}}\right\}$. So from dominated convergence we obtain that this term converges to $\int_{\left\{w<\frac{1}{\sqrt{e}}\right\}} w^{2} \log |w|$ as $R \rightarrow \infty$.

We now prove the liminf-inequality: Suppose $w_{\gamma} \rightarrow w \in \mathcal{A}$ in $L^{2}$ and without loss of generality assume that $J_{\gamma}\left(w_{\gamma}\right) \leq C$ for some constant $C$. So $w_{\gamma} \in \mathcal{A}$ for all $\gamma$, whence $\left\|w_{\gamma}\right\|_{L^{2}} \equiv 1=\|w\|_{L^{2}}$ and thus $w_{\gamma} \rightarrow w$ in $L^{2}$. By (5.6) and (5.5)

$$
\begin{aligned}
C & \geq J_{\gamma}\left(w_{\gamma}\right) \geq \frac{1}{2} \int_{\mathbb{R}^{n}}\left|\nabla w_{\gamma}\right|^{2}-c_{\gamma} D \int_{\mathbb{R}^{n}}\left|w_{\gamma}\right|^{2} \log \left|w_{\gamma}\right| \\
& \geq \frac{1}{4} \int_{\mathbb{R}^{n}}\left|\nabla w_{\gamma}\right|^{2}+\frac{c_{\gamma} D}{2} n\left(1+\frac{\log \pi}{2}-\frac{\log \left(2 c_{\gamma} D\right)}{2}\right),
\end{aligned}
$$

and so $\left(w_{\gamma}\right)$ is bounded in $H^{1}$ and $\nabla w_{\gamma} \rightarrow \nabla w$ in $L^{2}$.

For fixed $\varepsilon>0$ let $g_{\varepsilon}(t):=\min \left\{\max \left\{-\frac{1}{\varepsilon} t^{2}, t^{2} \log t\right\}, \frac{1}{\varepsilon}\right\}$. Since $\left(w_{\gamma}\right)$ is bounded in $L^{2^{*}}, \chi_{\left\{\left|w_{\gamma}\right| \geq 1\right\}}\left|w_{\gamma}\right|^{2}|\log | w_{\gamma}||$ is bounded in $L^{\eta}$ for some $\eta>1$ and we obtain

$$
\begin{aligned}
\liminf _{\gamma \rightarrow 2} J_{\gamma}\left(w_{\gamma}\right) & \geq \frac{1}{2} \int_{\mathbb{R}^{n}}|\nabla w|^{2}-D \limsup _{\gamma \rightarrow 2} \int_{\mathbb{R}^{n}}\left|w_{\gamma}\right|^{2} \log \left|w_{\gamma}\right| \\
& \geq \frac{1}{2} \int_{\mathbb{R}^{n}}|\nabla w|^{2}-D \limsup _{\gamma \rightarrow 2} \int_{\mathbb{R}^{n}} g_{\varepsilon}\left(\left|w_{\gamma}\right|\right)-\rho(\varepsilon) \\
& =\frac{1}{2} \int_{\mathbb{R}^{n}}|\nabla w|^{2}-D \int_{\mathbb{R}^{n}} g_{\varepsilon}(|w|)-\rho(\varepsilon)
\end{aligned}
$$

for some $\rho(\varepsilon) \rightarrow 0$ as $\varepsilon \rightarrow 0$. Now by the monotone convergence theorem, applied on $\{|w| \geq 1\}$ and $\{|w|<1\}$ separately, sending $\varepsilon \rightarrow 0$ we see that indeed $\liminf _{\gamma \rightarrow 2} J_{\gamma}\left(w_{\gamma}\right) \geq J(w)$.

In order to prove the last statement of the proposition, suppose $\left(w_{\gamma}\right)$ is an arbitrary recovery sequence. Then similarly as in (5.8) we find

$$
\begin{aligned}
\frac{1}{2} \int_{\mathbb{R}^{n}}|\nabla w|^{2}-D \int_{\mathbb{R}^{n}}|w|^{2} \log |w| & =\limsup _{\gamma \rightarrow 2} J_{\gamma}\left(w_{\gamma}\right) \\
& \geq \limsup _{\gamma \rightarrow 2} \frac{1}{2} \int_{\mathbb{R}^{n}}\left|\nabla w_{\gamma}\right|^{2}-D \limsup _{\gamma \rightarrow 2} \int_{\mathbb{R}^{n}} w_{\gamma}^{2} \log w_{\gamma} \\
& \geq \limsup _{\gamma \rightarrow 2} \frac{1}{2} \int_{\mathbb{R}^{n}}\left|\nabla w_{\gamma}\right|^{2}-D \int_{\mathbb{R}^{n}}|w|^{2} \log |w|,
\end{aligned}
$$

and so $\left\|\nabla w_{\gamma}\right\|_{L^{2}} \rightarrow\|\nabla w\|_{L^{2}}$. But then $\nabla w_{\gamma} \rightarrow \nabla w$ in $L^{2}$ since $\nabla w_{\gamma} \rightarrow \nabla w$, and the claim follows.

As noted before, the minimizers of $J_{\gamma}$ are the rescaled minimizers of $I_{\gamma}$, so there is a (unique) radially decreasing minimizer $w_{\gamma}$ of $J_{\gamma}$. We need the following mild equicoercivity result.

Proposition 5.5. Let $\gamma_{k} \rightarrow 2$ and $\left(w_{\gamma_{k}}\right)$ be a sequence of radially decreasing minimizers of $J_{\gamma}$. Then there is a subsequence (not relabeled) of $\left(w_{\gamma_{k}}\right)$ that converges to some $w \in \mathcal{A}$ strongly in $L^{2}$ and weakly in $H^{1}$.

Proof. We follow the strategy of the proof of Lemma 2.5. Let $\gamma_{k} \rightarrow 2$. As seen in the proof of Proposition 5.4, $\left\|w_{\gamma_{k}}\right\|_{H^{1}}$ can be estimated in terms of $J_{\gamma_{k}}\left(w_{\gamma_{k}}\right)$, so we may assume that $w_{k}:=w_{\gamma_{k}} \rightarrow w$ for some $w \in H^{1}$. It remains to show that $\|w\|_{L^{2}} \geq 1$. 
Assuming this were not the case, similarly as in the proof of Lemma 2.5 we find some fixed $\varepsilon>0$ such that for each $\delta>0$ there exists $R>0$ and a cut-off function $\theta: \mathbb{R}^{n} \rightarrow[0,1]$ with $\theta \equiv 1$ on $B_{R-1}, \theta \equiv 0$ on $\mathbb{R}^{n} \backslash B_{R}$, $|\nabla \theta|$ bounded independently of $R$ and

$$
\left\|\chi_{\mathbb{R}^{n} \backslash B_{R}} w_{k}\right\|_{L^{2}} \geq \varepsilon \text { for all } k \geq k_{0}(\delta), \quad\left|w_{k}\right| \leq \delta \text { on } \mathbb{R}^{n} \backslash B_{R-1}
$$

Now if $\delta \leq \frac{1}{\sqrt{e}}$, then $-w_{k}^{2} \log w_{k} \geq-\left(\theta w_{k}\right)^{2} \log \left(\theta w_{k}\right)$ on $B_{R} \backslash B_{R-1}$. Also note that $\left|\nabla\left(\theta w_{k}\right)\right|^{2} \leq 2\left|\nabla w_{k}\right|^{2}+$ $C w_{k}^{2}$. So

$$
\frac{1}{2} \int_{B_{R}}\left|\nabla w_{k}\right|^{2}-D c_{\gamma_{k}} \int_{B_{R}} w_{k}^{2} \log \left|w_{k}\right| \geq \frac{1}{4} \int_{B_{R}}\left|\nabla\left(\theta w_{k}\right)\right|^{2}-D c_{\gamma_{k}} \int_{B_{R}}\left(\theta w_{k}\right)^{2} \log \left|\theta w_{k}\right|-C
$$

and thus by (5.6) and the logarithmic Sobolev inequality (applied to $\frac{\theta w_{k}}{\left\|\theta w_{k}\right\|_{L^{2}}}$ )

$$
\begin{aligned}
J_{\gamma_{k}}\left(w_{k}\right) & \geq \frac{1}{2} \int_{B_{R}}\left|\nabla w_{k}\right|^{2}-D c_{\gamma_{k}} \int_{B_{R}} w_{k}^{2} \log \left|w_{k}\right|+D c_{\gamma_{k}} \int_{\mathbb{R}^{n} \backslash B_{R}} \frac{w_{k}^{\gamma_{k}}-w_{k}^{2}}{2-\gamma_{k}} \\
& \geq-C+c_{\gamma_{k}} \int_{\mathbb{R}^{n} \backslash B_{R}} \frac{w_{k}^{\gamma_{k}}-w_{k}^{2}}{2-\gamma_{k}}
\end{aligned}
$$

Now as in the proof of Lemma 2.5 by minimizing $\int v^{\gamma}$ for fixed $\int v^{2}$ under the constraint $0 \leq v \leq \delta$, we find that this implies

$$
J_{\gamma_{k}}\left(w_{\gamma_{k}}\right) \geq D c_{\gamma_{k}} \frac{\delta^{\gamma_{k}-2}-1}{2-\gamma_{k}}\left\|\chi_{\mathbb{R}^{n} \backslash B_{R}} w_{k}\right\|_{L^{2}}^{2}-C \geq D c_{\gamma_{k}} \frac{\delta^{\gamma_{k}-2}-1}{2-\gamma_{k}} \varepsilon^{2}-C
$$

for all $k \geq k_{0}$. Taking the $\lim \sup _{k \rightarrow \infty}$, we obtain $C \geq-\varepsilon^{2} \log \delta$. This leads to a contradiction when $\delta \rightarrow 0$.

Proof of Theorem 5.3. Using Propositions 5.4 and 5.5, as in Corollary 2.6 we see that every sequence $\left(w_{\gamma_{k}}\right)$ has a subsequence converging strongly in $L^{2}$ and weakly in $H^{1}$ to a radially decreasing minimizer of $J$. Since this minimizer is uniquely given by

$$
w(x)=\left(\frac{D}{2 \pi}\right)^{\frac{n}{2}} \mathrm{e}^{-\frac{D x^{2}}{2}}
$$

by the logarithmic Sobolev inequality (5.5), the whole sequence $\left(w_{\gamma}\right)$ converges to $w$. But then $\left(w_{\gamma}\right)$ is a recovery sequence for $w$ and $w_{\gamma} \rightarrow w$ in $H^{1}$ by Proposition 5.4. Furthermore,

$$
\frac{I_{\gamma, D}\left(u_{\gamma, D}\right)-D c_{\gamma}}{2-\gamma}=J_{\gamma}\left(w_{\gamma}\right) \rightarrow J(w)=\frac{D n}{2}\left(1+\frac{\log \pi-\log D}{2}\right) .
$$

The proof of the remaining part of the theorem on the limiting behavior of $u_{\gamma, \frac{D}{2-\gamma}}$ follows exactly along the same lines, when $c_{\gamma}$ in the definition of $J_{\gamma}$ is replaced by 1 .

Acknowledgements. I would like to thank Wolfgang König for pointing out this problem to me and for interesting discussions on the subject. 


\section{REFERENCES}

[1] H. Berestycki and P.L. Lions, Nonlinear scalar field equations I. Existence of a ground state. Arch. Rational Mech. Anal. 82 (1983) 313-345.

[2] M. Biskup and W. König, Long-time tails in the parabolic Anderson model with bounded potential. Ann. Probab. 29 (2001) 636-682.

[3] A. Braides, Г-Convergence for Beginners. Oxford University Press, Oxford, UK (2002).

[4] J.E. Brother and W.P. Ziemer, Minimal rearrangements of Sobolev functions. J. Reine Angew. Math. 384 (1988) $153-179$.

[5] C.C. Chen and C.S. Lin, Uniqueness of the ground state solutions of $\Delta u+f(u)=0$ in $\mathbb{R}^{n}, n \geq 3$. Comm. Partial Diff. Eq. 16 (1991) 1549-1572.

[6] C. Cortazar, M. Elgueta and P. Felmer, On a semilinear elliptic problem in $\mathbb{R}^{N}$ with a non-Lipschitzian nonlinearity. Adv. Diff. Eq. 1 (1996) 199-218.

[7] C. Cortazar, M. Elgueta and P. Felmer, Uniqueness of positive solutions of $\Delta u+f(u)=0$ in $\mathbb{R}^{N}, N \geq 3$. Arch. Rational Mech. Anal. 142 (1998) 127-141.

[8] J. Gärtner and S.A. Molchanov, Parabolic problems for the Anderson model. I. Intermittency and related topics. Comm. Math. Phys. 132 (1990) 613-655.

[9] W. König, Große Abweichungen, Techniken und Anwendungen. Vorlesungsskript Universität Leipzig, Germany (2006).

[10] M.K. Kwong, Uniqueness of positive solutions of $\Delta u-u+u^{p}=0$ in $\mathbb{R}^{n}$. Arch. Rational Mech. Anal. 105 (1989) $243-266$.

[11] E.H. Lieb and M. Loss, Analysis, AMS Graduate Studies 14. Second edition, Providence, USA (2001).

[12] P. Pucci, M. García-Huidobro, R. Manásevich and J. Serrin, Qualitative properties of ground states for singular elliptic equations with weights. Ann. Mat. Pura Appl. 185 (2006) 205-243. 